\title{
RDUS
}

Revue de DROIT

UNIVERSITÉ DE SHERBROOKE

Titre : $\quad$ À LA RECHERCHE D'UN STATUT JURIDIQUE POUR LES MIGRANTS ENVIRONNEMENTAUX TRANSFRONTALIERS : LA PROBLÉMATIQUE DE LA NOTION DE RÉFUGIÉ

Auteur(s): $\quad$ Pierre-François MERCURE

Revue : $\quad$ RDUS, 2006-2007, volume 37, numéro 1

Pages: $\quad 1-39$

ISSN : $\quad 0317-9656$

Éditeur : $\quad$ Université de Sherbrooke. Faculté de droit.

URI : $\quad$ http://hdl.handle.net/11143/11826

DOI : $\quad$ https://doi.org/10.17118/11143/11826 
Page vide laissée intentionnellement. 
ARTICLE

\section{À LA RECHERCHE D'UN STATUT JURIDIQUE POUR LES MIGRANTS ENVIRONNEMENTAUX TRANSFRONTALIERS : LA PROBLÉMATIQUE DE LA NOTION DE RÉFUGIÉ}

par Pierre-François MERCURE ${ }^{\dagger}$

Le nombre de personnes contraintes de se déplacer à la suite de désastres environnementaux augmentera significativement au cours des prochaines décennies. Ce sont principalement les modifications lentes de l'environnement, notamment les changements climatiques, qui seront à l'origine des mouvements de populations.

L'analyse qui suit tente de démontrer que les solutions au problème des migrants environnementaux transfrontaliers ne peuvent être envisagées sans réviser le concept de l'État-nation qui sert d'assise aux relations internationales. Par ailleurs, elles ne sont pas praticables sans que l'économie mondiale soit adaptée à la gestion des préoccupations écologiques globales. Ces avenues constituent des préalables incontournables à l'élaboration d'une stratégie intégrée afin d'encadrer les vagues croissantes de migrants environnementaux. L'étude expose aussi que ces derniers peuvent difficilement invoquer, avec succès, le statut de réfugié afin de demander l'asile politique. Il serait préférable d'envisager l'élaboration d'un cadre normatif spécifique à leur situation. Il est alors possible, soit de modifier les conventions existantes, soit d'élaborer une convention adaptée au contexte particulier des personnes victimes de modifications de l'environnement.

The coming decades will witness a significant increase in the number of displaced persons due to environmental disasters. Slowly evolving environmental changes, notably climate changes, will provoke the shifting of populations.

The present study seeks to establish that one cannot contemplate resolution of the problem of transborder environmental migration without reexamining the concept of the Nation State, which serves as the basis of international relations. Moreover, the solutions proposed cannot be applied unless the world economy is adapted so as to address worldwide environmental concerns. These adaptations are unavoidable prerequisites to the elaboration of an integrated strategy intended to cope with the growing waves of environmental migrants. This article also points out that these migrants cannot successfully invoke refugee status in order to claim political asylum. It is preferable to set out a specific normative framework for people in this situation. It would thus be possible to either modify existing conventions or to draft a new convention adapted to the particular situation of victims of environmental changes.

Ce texte constitue une version remaniée d'une communication présentée le 13 novembre 2005 à la Faculté de droit de l'Université d'Ain Chams au Caire, dans le cadre d'un colloque organisé par le Réseau Droits de l'homme de l'Association des universités francophones. L'auteur remercie le Fonds québécois de la recherche sur la société et la culture de son soutien financier pour la présentation de la communication et la préparation de ce texte.

$\dagger \quad$ Professeur à la Faculté de droit de l'Université de Sherbrooke. 


\section{SOMMAIRE}

Introduction

Première partie : L'inadéquation des concepts traditionnels des relations internationales aux migrations environnementales....

A) La réévaluation des conjonctures politique et économique actuelles.

a) La nécessité de redéfinir la portée du concept de l'État-

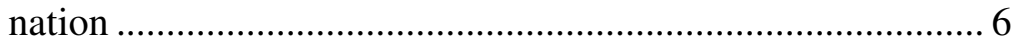

b) L'adaptation du système économique 8

B) Les contraintes inhérentes à l'application de la notion de réfugié

a) La signification de la crainte fondée d'être persécuté........... 13

i) Les faits de l'affaire Sarei v. Rio Tinto ..................... 15

ii) Le contenu des critères subjectifs et objectifs dans une situation de dégradation de l'environnement, à la lumière de l'affaire Sarei v. Rio Tinto.

b) Les limites à considérer la dégradation de l'environnement comme une forme de persécution

Deuxième partie : La définition d'un cadre normatif

international 22

A) Les amendements au droit positif des réfugiés ............................... 22

a) La reconnaissance du droit à la qualité de l'environnement .. 22

b) La pauvreté comme motif de persécution, dans un contexte de modification de l'environnement 26

i) La difficulté d'attribuer à la race un motif de persécution environnementale .................................. 26

ii) L'appartenance au groupe social des démunis .......... 28

B) L'adoption d'une convention portant sur les migrations environnementales

a) La conciliation des objectifs de la convention avec les particularismes régionaux

b) Les principes sous-jacents à la convention, selon Gregory S. McCue 35 
À la recherche d'un statut juridique pour
(2006) 37 R.D.U.S. $\quad$ les migrants environnementaux transfrontalier

la problématique de la notion de réfugié

\section{Introduction}

Les déplacements de personnes, résultant d'altérations de l'environnement, augmentent d'année en année ${ }^{1}$. Ils pourraient connaître un accroissement significatif au cours des prochaines décennies en raison de la recrudescence des catastrophes industrielles, mais aussi à cause des effets engendrés par les modifications lentes de l'environnement, notamment ceux résultants des changements climatiques ${ }^{2}$. Les conséquences de ces derniers ont comme caractéristique de restreindre significativement les possibilités de retour dans le pays d'origine. Les résidants des micros États insulaires du Pacifique, potentiellement submergés par l'augmentation du niveau des océans consécutive au réchauffement de la planète, pourraient être confrontés à une situation de cette nature ${ }^{3}$.

Environ 144 millions de personnes subissent, chaque année, les effets directs des modifications de leur milieu ${ }^{4}$. De ce nombre, 25 millions sont contraintes de déménager pour survivre ${ }^{5}$. Le Haut Commissariat des Nations Unies pour les réfugiés [HCNUR] estime que 3 millions de migrants environnementaux s'ajoutent annuellement à ceux existants ${ }^{6}$.

Les déplacements de personnes se produisent principalement à l'intérieur des frontières des États dans lesquels l'environnement est perturbé. L'ampleur des mutations au sein des écosystèmes, occasionnées par les modifications du climat, est cependant susceptible d'augmenter le nombre des

1. Ils résultent de catastrophes naturelles, de la dégradation progressive de l'environnement, du développement, d'accidents industriels et des vestiges de la guerre. David Keane, «The environmental causes and consequences of migration : a search for the meaning of environmental refugees» (2004) 16 Geo. Int'l Envtl. L. Rev. 209 à la p. 211.

2. Inondations, désertification, famines, etc. En 1998, pour la première fois depuis que des statistiques sont compilées à ce sujet, les désastres naturels ont été responsables de déplacements de population supérieurs à ceux causés par les conflits armés. D. Keane, ibid.

3. Caroline Touzin, «Tuvalu : menacé de disparaître, Futurs réfugiés écologiques» La Presse, (14 février 2005).

4. D. Keane, supra note 1.

5. Fédération internationale de la Croix-Rouge et du Croissant-Rouge, Rapport sur les désastres dans le monde, Chapitre 5 (2003); Jessica B. Cooper, «Environmental refugees : meeting the requirements of the refugee definition» (1998) 6 N.Y.U. Envtl. L.J. 480 à la p. 485.

6. D. Keane, supra note 1 à la p. 218. 
À la recherche d'un statut juridique pour

les migrants environnementaux transfrontaliers:

la problématique de la notion de réfugié

migrations transfrontalières. C'est dans une perspective internationale, c'està-dire celle du franchissement d'une frontière par un individu, que sera étudiée la question des migrants environnementaux. Les aspects juridiques des mouvements intra étatiques de populations ne seront pas abordés.

Les contextes politique et économique sont inadaptés à la problématique des migrations transfrontalières découlant des altérations de l'environnement. Le concept de l'État-nation à économie de marché, qui constitue le fondement du droit international contemporain, devrait être revu. Il est, en effet, la cause principale de l'existence, voire de l'accélération de la production des réfugiés, dans le sens juridique classique attribué à ce terme. Le système économique mondial devrait aussi être modifié, afin que les coûts associés aux migrations environnementales transfrontalières soient assumés par les acteurs sociaux qui les provoquent, proportionnellement à leur part de responsabilité dans les facteurs qui en sont à l'origine.

L'encadrement normatif des déplacements de populations résultant des modifications du milieu, devra être arrimé avec celui retenu pour assurer la gestion des mécanismes de stabilisation du climat, puisque les premiers dépendront des deuxièmes. La refonte des modes de gouvernance internationale qu'impose la stabilisation du climat implique, par conséquent, une révision des processus décisionnels pour traiter les mouvements transfrontaliers de populations. La question des changements climatiques servira donc fréquemment à illustrer les concepts présentés dans l'étude.

Le système juridique international a été enrichi de dispositions afin de fournir une assistance humanitaire aux victimes de catastrophes environnementales ${ }^{7}$. Il est cependant incapable de répondre à l'accroissement du nombre des migrants environnementaux.

L'expression réfugiés environnementaux a été créée en 1985 par le juriste El-Hinnawi qui les définit comme «des personnes qui ont été forcées de quitter leur habitat traditionnel, temporairement ou de façon permanente, à

7. À titre d'exemple, consulter la Résolution 43/131 de l'Assemblée générale des Nations Unies relative à l'assistance humanitaire aux victimes de catastrophes naturelles et situations d'urgence du même ordre, adoptée le 8 décembre 1988, dans P.M. Dupuy, Les grands textes de droit international public, 2e éd., Paris, Dalloz, 2000 à la p. 121. 
À la recherche d'un statut juridique pour
(2006) 37 R.D.U.S. $\quad \begin{gathered}\text { les migrants environnementaux transfrontaliers } \\ \text { la problématique de la notion de réfugié }\end{gathered}$

cause d'une importante perturbation de l'environnement (d'origine naturelle ou anthropique) qui menace leur existence et/ou affecte sérieusement leur qualité de vie» ${ }^{8}$. Le droit international n'apporte pas de solutions adaptées aux mouvements de populations, consécutifs aux modifications de l'environnement. La notion de réfugié, énoncée dans les accords universels, notamment la Convention relative au statut des réfugiés (1951) [Convention de 1951] ${ }^{9}$ et le Protocole relatif au statut des réfugiés (1967) [Protocole de 1967] ${ }^{10}$ et dans les accords régionaux, telle la Convention de l'Organisation de l'unité africaine régissant les aspects propres aux problèmes des réfugiés en Afrique (1969) $)^{11}$, est difficilement applicable aux migrations transfrontalières de personnes.

Les contraintes mentionnées obligent à situer la problématique des migrants environnementaux dans le contexte d'un cadre conventionnel international adapté.

La première partie du travail analyse les difficultés rencontrées afin d'appliquer les concepts traditionnels des relations internationales aux migrations environnementales. La deuxième partie est, quant à elle, consacrée à la détermination d'un cadre normatif qui concerne ce segment de la population.

\section{Première partie : L’inadéquation des concepts traditionnels des relations internationales aux migrations environnementales}

Le système traditionnel des relations internationales, qui s'articule autour du concept de l'État-nation de type libéral, devrait être révisé. Il est donc nécessaire de procéder à la réévaluation des conjonctures politique et économique actuelles dans lesquelles la communauté des États évolue (A).

8. Rapporté dans D. Keane, supra note 1 à la p. 210.

9. $\quad$ Convention relative au statut des réfugiés, adoptée le 28 juillet 1951. 189 R.T.N.U. 151 : R.T.C. 1969 , no 6.

10. Protocole relatif au statut des réfugiés, adopté le 31 janvier 1967, 606 R.T.N.U. 267; R.T.C. 1969, no 29.

11. Convention de l'Organisation de l'unité africaine régissant les aspects propres aux problèmes des réfugiés en Afrique, 10 septembre 1969, 1001 R.T.N.U. 45 [Convention de l'OUA]; Organisation de l'unité africaine (ci-après «OUA»). Infra note 91. 
À la recherche d'un statut juridique pour

les migrants environnementaux transfrontaliers:

la problématique de la notion de réfugié

Depuis 1975, les personnes déplacées font partie du mandat du $\mathrm{HCNUR}^{12}$. Ce dernier définit ainsi le terme «réfugié»: toute personne ou groupe de personnes qui, s'ils avaient franchi une frontière internationale seraient des réfugiés ${ }^{13}$. Les migrants environnementaux sont-ils inclus dans cette catégorie? La réponse à cette question requiert l'étude des contraintes inhérentes à l'application de la notion de «réfugié» aux migrations environnementales (B).

\section{A) La réévaluation des conjonctures politique et économique actuelles}

Une réflexion portant sur la gestion des migrations environnementales implique de repenser l'organisation des relations internationales et concomitamment, celle de leurs fondements économiques. Il est donc pertinent d'envisager la redéfinition de la portée du concept de l'État-nation (a) et de s'interroger sur la réforme souhaitée du système économique (b).

\section{a) La nécessité de redéfinir la portée du concept de l'État-nation}

Les déplacements de populations constituent un problème global lorsqu'ils résultent des changements climatiques. Les structures de l'État, mais aussi celles des organisations internationales nées dans l'après-guerre, sont inadaptées à la résolution de ce type d'enjeux internationaux. En effet :

L'agenda post guerre froide est bien celui de la reconnaissance des problèmes globaux et des difficultés de la communauté internationale à trouver les procédures, les institutions, mais aussi les consensus pour les traiter ${ }^{14}$.

C'est dans cette perspective que doivent être situées les migrations environnementales transfrontalières, pour lesquelles il n'existe aucune procédure, institution ou consensus. Cette défaillance est en grande partie attribuable au fait que les réfugiés sont, au sens large, des sous-produits du

12. Voir Le HCNUR en bref, en ligne : http://www.unhcr.fr (date d'accès 2 octobre 2006).

13. D. Keane, supra note 1 à la p. 217.

14. I. Kaul et al., Les biens publics mondiaux, La coopération internationale au XXIe siècle, Paris, Economica, 2002 à la p. XII. 
À la recherche d'un statut juridique pour
(2006) 37 R.D.U.S. $\quad \begin{gathered}\text { les migrants environnementaux transfrontaliers } \\ \text { la problématique de la notion de réfugié }\end{gathered}$

concept de l'État-nation, né au $16^{\mathrm{e}}$ siècle $^{15}$, et qui s'appuie sur le principe de la souveraineté. Ce concept a été élaboré à partir d'une représentation erronée du monde, selon laquelle l'État devait servir de cadre juridique à une seule nation. Cette vision théorique ne traduisait pas la réalité de l'époque, où les peuples étaient déjà «entremêlés». Elle n'a plus rien à voir avec le monde contemporain. Ce dernier est, en effet, constitué d'États aux visages de plus en plus métissés et multiethniques, à cause de la mobilité croissante des personnes, que la mondialisation accentue.

Dans un tel contexte, les conflits sont inéluctables et l'apparition de réfugiés l'est aussi, puisqu'il n'existe aucune adéquation entre les nations et la composante essentielle de l'État que constituent ses frontières. Des factions nationales s'opposent alors entre elles, pour obtenir le contrôle de l'État. Malgré ce phénomène, le droit considère la question des réfugiés comme une anomalie, plutôt qu'une partie intégrante du système international ${ }^{16}$. Les tensions qui découlent de l'écart existant entre la réalité et le concept de l'État-nation sont responsables de la production des réfugiés et ont donné naissance aux théories politiques qui leurs sont applicables. Ces dernières s'appuient sur la notion de la «persécution» des personnes qui fuient un territoire $^{17}$. Elles expliquent les contraintes des traités et structures disponibles, afin de porter aide aux réfugiés.

Les migrants environnementaux sont tout aussi inévitables que les réfugiés politiques, car le système international qui entend gérer les problématiques écologiques globales se fonde sur le concept traditionnel de l'État-nation jouissant d'une souveraineté quasi-absolue. Cela fait dire à un auteur :

This concept would coincide with reality if borders were, in fact, impenetrable, or if nature was always predictable. However, as long as nature disastrously combines with human mismanagement,

\footnotetext{
15. Q.D. Nguyen, P. Daillier et A. Pellet, Droit international public, 7e éd., Paris, L.G.D.J., 2002 à la p. 51.

16. Gregory S. McCue, «Environmental refugees : applying international environmental law to involuntary migration » (1993) 6 Geo. Int'l Envtl. L. Rev. 151. p. 168.

17. Ibid. à la p. 169.
} 
rational actors all over the globe will flee when they can, rather than remain and $\mathrm{die}^{18}$.

La remise en question du concept de l'État-nation a pour cadre de réflexion, la recherche d'un ordre international s'articulant autour d'une vision multilatérale, limitative des prérogatives de la souveraineté. L'objectif est de renforcer les capacités des États nations à faire face à l'interdépendance mondiale ${ }^{19}$. Les préoccupations globales contemporaines, qu'elles soient économiques, sociales ou environnementales, requièrent une modification en profondeur de la gouvernance politique de l'État. Elles ne seront solutionnées que par un ajustement des structures macro-économiques, non seulement des États, mais du système international dans son intégralité.

La résolution du problème des migrations environnementales doit être située dans celle plus large de la gestion économique des problèmes écologiques globaux. C'est à partir de commentaires relatifs à l'élaboration d'un contexte économique propice à ralentir, dans un premier temps, puis à résoudre, dans un deuxième temps, les changements anthropiques du climat, que sera envisagée la question des migrants environnementaux.

\section{b) L'adaptation du système économique}

L'augmentation des coûts financiers associés aux migrations transfrontalières oblige à situer la problématique sous l'angle du concept des biens publics mondiaux développé par les Nations Unies. L'objectif de ce dernier, appliqué aux problèmes environnementaux globaux, est de faire payer les responsables des conséquences de ceux-ci, en amont de leurs opérations. Le système économique mondial devrait, par conséquent, être revu, afin que les coûts associés aux migrations environnementales transfrontalières soient assumés par ceux qui les causent, proportionnellement à leur part de responsabilité dans les facteurs qui en sont à l'origine.

L'hypothèse de l'augmentation de la part contributive du réchauffement planétaire à l'accroissement annuel proportionnel des migrants

18. Ibid. à la p. 170 .

19. Les biens publics mondiaux, supra note 14 à la p. 15. 
À la recherche d'un statut juridique pour
(2006) 37 R.D.U.S. $\quad$ les migrants environnementaux transfrontalier

la problématique de la notion de réfugié

environnementaux semble se confirmer dans la communauté scientifique ${ }^{20}$. L'économie des migrations environnementales ne peut donc être dissociée de celle des modifications du climat. Il convient alors de commenter cette dernière, qui a été l'objet de plusieurs études, afin de comprendre la première, encore ignorée par la science économique.

Comme beaucoup de problèmes globaux, les changements climatiques trouvent, en grande partie, leur origine dans le dysfonctionnement de l'économie mondiale. Cette dernière ne tiendrait pas compte des «externalités» ${ }^{21}$, induites à chacune des étapes de production des gaz à effet de serre. L'«externalité» peut-être définie comme suit :

Phénomène qui se produit quand un individu ou une entreprise agit, mais ne supporte pas tous les coûts (externalités négatives) ou ne reçoit pas tous les avantages (externalités positives) de son action ${ }^{22}$.

L'émission des gaz à effet de serre prend sa source dans le fait que les acteurs sociaux: individus, entreprises et États, n'assument pas leurs responsabilités économiques respectives face à ce phénomène. D'un autre côté, ces acteurs ne reçoivent pas tous les avantages induits par celui-ci. Ainsi, la pollution de l'air causée par une entreprise constitue une «externalité» négative pour cette dernière, puisqu'elle ne supporte pas tous les coûts des dommages associés aux émanations nocives. D'un autre côté, l'installation d'équipements de captation des émissions polluantes constitue une «externalité» positive pour la même entreprise, dans un contexte où les incitatifs gouvernementaux de nature économique ne compensent pas les investissements réalisés. Ainsi en est-il, par exemple, d'abattements fiscaux ou de subventions inadéquats.

20. Selon Philippe Le Prestre, «les changements climatiques menacent de redistribuer les populations d'une manière et avec des conséquences difficiles à prévoir». Philippe Le Prestre, Protection de l'environnement et relations internationales, Les défis de l'écopolitique mondiale, Paris, Armand Colin, 2005 à la p. 390.

21. «Phénomène qui se produit quand un individu ou une entreprise agit, mais ne supporte pas tous les coûts (externalités négatives) ou ne reçoit pas tous les avantages (externalités positives) de son action», supra à la p. 264. 
À la recherche d'un statut juridique pour

les migrants environnementaux transfrontaliers: (2006) 37 R.D.U.S.

la problématique de la notion de réfugié

L'une des «externalités» négatives des émissions de gaz à effet de serre est constituée par le coût associé aux migrations environnementales. Les États devraient normalement assumer la proportion de ce dernier, qui correspond à leur part de responsabilité dans le réchauffement planétaire. À titre d'exemple, environ les deux tiers des coûts totaux des changements climatiques devraient être répartis entre les acteurs sociaux concernés des pays développés, puisqu'ils sont à l'origine d'une telle proportion des émissions totales de gaz à effet de serre ${ }^{23}$. Ils devraient donc supporter un pourcentage équivalent aux coûts associés aux migrations de personnes.

L'instauration de mécanismes visant l'«internalisation» des «externalités» incombe aux États. Les économistes considèrent que c'est l'irresponsabilité des États, à ce niveau, qui causerait les «maux publics mondiaux» ${ }^{24}$, tels le terrorisme, les guerres, les modifications climatiques, etc. Les migrations environnementales sont, sans contredit, comprises dans cette catégorie.

C'est parce que les États ne gèrent pas convenablement le milieu ambiant que des migrations environnementales se produisent. La négligence des États à réglementer les émissions de gaz à effet de serre engendre des catastrophes environnementales et leur laxisme à imposer aux entreprises des normes de gestion, au sens large, cause des accidents industriels, dont les conséquences sur l'environnement sont, aussi, très importantes. Tel fut le cas, par exemple, de la pollution radioactive engendrée par l'explosion de la centrale nucléaire de Tchernobyl. Les États ont l'obligation d'adopter, à la source, des mesures afin de prévenir et de contenir les maux publics mondiaux à l'intérieur même de leurs frontières :

23. Laramée V., Martin-Ferrari D. et Bernard M., site de l'AED «Qui émet quoi et combien?» (2 novembre 2005) en ligne : <http://www.aed-dmf.com/climat/tous/ climattousemission.thm>. Voir aussi : J.M. Manicore, «Comment évolue maintenant les émissions de gaz à effet de serre?» (2 février 2005) en ligne : $<$ http://www.manicore.com/documentation/serre/GES.html.

24. Comme le rapportent I. Kaul et al.: «les biens publics génèrent d'importantes externalités, positives ou négatives, auquel cas on parle souvent de «mal public», Les biens publics mondiaux», supra note 14 à la p. 3 . 
Cela exigera en particulier des mesures pour «internaliser les externalités» - i.e. pour résoudre les problèmes à la source avant qu'ils ne débordent des frontières ${ }^{25}$ (notre soulignement).

L'adoption de dispositions semblables permettrait aux États d'assumer les responsabilités qui leur reviennent dans la gestion des migrations environnementales transfrontalières. Leurs devoirs, à cet égard, comportent deux volets indissociables :1) la responsabilité des «externalités» qui prennent leur source à l'intérieur de leurs frontières, et 2) la responsabilité (notamment financière) des retombées transfrontalières générées par leurs ressortissants ${ }^{26}$.

La remise en question de la gouvernance mondiale ne saurait être complète, sans parallèlement évaluer la pertinence d'appliquer aux migrants environnementaux transfrontaliers, les mécanismes du droit positif des réfugiés.

\section{B) Les contraintes inhérentes à l'application de la notion de réfugié}

Le HCNUR a été créé le 14 décembre 1950 par l'Assemblée générale des Nations Unies avec le mandat de coordonner l'action internationale pour la protection des réfugiés ${ }^{27}$ et de chercher des solutions au problème des réfugiés. La détermination des paramètres qui font d'une personne un réfugié relève du droit international, plus précisément de la Convention de 1951 et du Protocole de 1967 relatifs au statut de réfugiés ${ }^{28}$ ou d'accords régionaux ${ }^{29}$. Le

25. Ibid. à la p. 12.

26. Ibid. aux pp. $14-15$.

27. Le préambule de la Convention de 1951 mentionne : «le Haut Commissaire des Nations Unies pour les réfugiés a pour tâche de veiller à l'application des conventions internationales qui assurent la protection des réfugiés (...)», supra note 9.

28. Convention de 1951, art. 1) A) 2), supra note 9; Protocole de 1967, art. 1) 2), supra note 10; Convention des Nations Unies sur le droit de la mer, 10 décembre 1982, Doc.NU A/CONF.62/122, R.T.N.U. 1833 (entrée en vigueur : 16 novembre 1994).

29. Mentionnons les documents suivants pour l'Amérique : Traité de droit pénal international, 23 janvier 1889, OEA/Ser.X/I; Accord sur l'extradition, 25 février 1981, OEA/Ser.A/36 (entrée en vigueur : 28 mars 1992); Convention sur l'asile, 20 février 1928, OEA/Ser.X/I (entrée en vigueur : 21 mai 1929); Convention sur l'asile politique, 26 décembre 1933, OEA/Ser.X/I (entrée ne vigueur : 28 mars 1935); Convention sur l'asile diplomatique, 28 mars 1954, OEA/Ser.A/9 (entrée en vigueur : 29 décembre 1954); Convention sur l'asile territorial, 28 mars 1954, OEA/Ser.A/10 (entrée en 
HCNUR, qui est responsable de l'application de la Convention de 1951 et du Protocole de 1967, est l'autorité compétente afin de déclarer que des personnes jouissent de la protection accordée par ces conventions ${ }^{30}$. Il veille donc à la sauvegarde des droits des réfugiés.

Le HCNUR a publié, en 1979, un Guide de procédures et critères à appliquer pour déterminer le statut de réfugié au regard de la convention de 1951 et du protocole de 1967 relatifs au statut des réfugiés [Guide] ${ }^{31}$. Il appartient à l'État administrant le territoire sur lequel la personne se trouve au moment où elle demande que le statut de réfugié lui soit reconnu, d'établir la procédure appropriée afin de lui conférer le statut de réfugié, en conformité avec, d'une part, la Convention de 1951 et le Protocole de 1967 et, d'autre part, les particularités constitutionnelles et administratives qui lui sont propres $^{32}$. La demande d'asile d'une personne doit être traitée en vertu des lois de l'État dans lequel la personne se trouve, mais ce sont la Convention de 1951 et le Protocole de 1967 qui prévoient l'étendue des obligations que les États doivent respecter à l'égard des réfugiés.

Le Protocole de 1967 définit le terme réfugié, comme suit :

Toute personne qui craignant avec raison d'être persécutée du fait de sa race, de sa religion, de sa nationalité, de son appartenance à un certain groupe social ou de ses opinions politiques, se trouve hors du pays dont elle a la nationalité et qui ne peut ou, du fait de cette crainte, ne veut se réclamer de la protection de ce pays $(. . .)^{33}$.

vigueur: 29 décembre 1954). Mentionnons le document suivant pour l'Afrique: Convention de l'Organisation de l'unité africaine régissant les aspects propres aux problèmes des réfugiés en Afrique, supra note 11 (entrée en vigueur : 20 juin 1974).

30. Statut du HCNUR, 14 décembre 1950. A. G. N. U. Rés. 428 (V), en ligne : <http://www.unhchr.ch/french/html/menu3/b/o_unhcr_fr.htm> (15 septembre 2005).

31. Le document est mis à jour régulièrement et la dernière version a été adoptée en 1992. Guide des procédures et critères à appliquer pour déterminer le statut de réfugié au regard de la convention de 1951 et du protocole de 1967 relatifs au statut des réfugiés Doc.UN HCR/1P/4FRE/REV.1 (1992) [Guide].

32. Guide, ibid., art. 189.

33. La suite de l'article se lit ainsi : «ou qui, si elle n'a pas de nationalité et se trouve hors du pays dans lequel elle avait sa résidence habituelle à la suite de tels événements, ne peut ou, en raison de ladite crainte, ne veut y retourner». L'art. 1, 2) du Protocole de 1967 réfère au terme réfugié de l'art. 1) A) 2) de la Convention de 1951. 
La plupart des spécialistes considèrent que les migrants environnementaux transfrontaliers ne pourraient être considérés comme des réfugiés ${ }^{34}$. Le droit international ne leur reconnaîtrait aucun statut juridique et les États n'auraient, par conséquent, aucune obligation à leur égard ${ }^{35}$. La définition de réfugié comporte plusieurs éléments. Certains de ceux-ci constituent des obstacles à l'attribution du statut de réfugié aux migrants environnementaux. Il s'agit: de la crainte fondée d'être persécuté et des motifs desquels découle la persécution, c'est-à-dire, la race, la religion, la nationalité, l'appartenance à un groupe social ou les opinions politiques ${ }^{36}$. Les commentaires sur ces deux aspects seront faits à travers une étude de la signification de l'expression «crainte fondée d'être persécuté» (a).

L'un des éléments fondamentaux du statut de réfugié est que la personne craigne pour son intégrité physique ou morale, si elle retourne dans le pays qu'elle a fui et que sa crainte est fondée. Puisque la dégradation de l'environnement est susceptible de constituer une atteinte à l'intégrité physique ou morale d'une personne, il est légitime de se poser la question afin de savoir si cette dernière peut fonder une crainte légitime, susceptible de rencontrer les critères de la définition de réfugié. Il sera ainsi constaté que la dégradation de l'environnement peut, dans des circonstances spécifiques, constituer une forme de persécution (b).

\section{a) La signification de la crainte fondée d'être persécuté}

Le premier élément de la définition du terme réfugié, la crainte fondée d'être persécuté, doit rencontrer un critère subjectif et un critère objectif. Pour le premier, la personne doit manifester un état d'esprit inspiré par la crainte ou la peur. Le deuxième critère s'analyse en se référant à l'expression «avec raison» qui indique que la crainte doit être fondée sur une situation objective, c'est-à-dire factuelle. Cette dernière implique, pour les autorités appelées à déterminer la qualité de réfugié, de procéder à une

34. Voir notamment D. Keane, supra note 1 à la p. 215 et J. B. Cooper, supra note 5 aux pp. 489-92.

35. D. Keane, ibid. à la p. 217.

36. Les deux autres conditions sont : «1) la personne est localisée à l'extérieur du pays de sa nationalité et 2) elle est incapable ou ne veut obtenir une protection de son propre pays ou elle n'a pas de nationalité et ne peut ou ne veut retourner dans le pays de sa résidence habituelle». Guide, supra note 31, art. 87-100. 
évaluation du contexte prévalant dans le pays que la personne fuit. L'élément subjectif sera déterminant afin de conférer le statut de réfugié à une personne, puisque la notion de crainte est subjective en $\mathrm{soi}^{37}$.

Il n'existe aucune définition unanimement acceptée du mot «persécution», mais il est admis qu'elle réfère toujours à une menace pour la vie ou la liberté fondée sur la race, la religion, la nationalité, l'appartenance à un groupe social et les opinions politiques ${ }^{38}$. Ces motifs constituent, selon le Guide, les seules causes valables de départ d'une personne, afin de la qualifier de réfugiée ${ }^{39}$.

La persécution doit nécessairement être le fait des autorités d'un pays, ou celui de groupes de la population qui ne se conforment pas aux lois de l'État ${ }^{40}$.

L'appréciation de l'élément subjectif requiert une évaluation de la personnalité $\mathrm{du}$ demandeur d'asile, étant donné que les réactions psychologiques des individus ne sont pas forcément identiques dans les mêmes circonstances ${ }^{41}$. Les autochtones, par exemple, qui, par leurs traditions et croyances religieuses entretiennent une relation intime avec le milieu ambiant, pourraient se voir accorder le statut de réfugié plus aisément que d'autres communautés humaines, dans l'éventualité où une modification de l'environnement constituerait un motif de persécution ${ }^{42}$. C'est la démonstration que fait un juriste américain en procédant à l'analyse de l'affaire Sarei v. Rio Tinto ${ }^{43}$.

Cette décision ne concerne pas une demande pour obtenir le statut de réfugié. Il s'agit d'un recours collectif afin que soient accordés des

37. Guide, ibid., art. 37-38.

38. Ibid., art. 51.

39. Ibid.

40. Ibid., art. 65 .

41. Ibid., art. 40.

42. Christopher M. Kozoll, «Poisoning the well : persecution, the environment, and refugee status», (2004) 15 Colo. J. Int'l Envtl. L. \& Pol'y 271 à la p. 280.

43. Sarei v. Rio Tinto 221 F. Supp.2d 1116 (C.D. Cal. 2002), en ligne : $<$ http://www.cacd/.uscourts.gov/CACD/RecentPubOp.nsf/> (9 janvier 2006). Pour un résumé des faits et une analyse détaillée de la décision, consulter C. M. Kozoll, ibid.à la p. 271 et ss. 
À la recherche d'un statut juridique pour
(2006) 37 R.D.U.S. $\quad \begin{gathered}\text { les migrants environnementaux transfrontaliers : } \\ \text { la problématique de la notion de réfugié }\end{gathered}$

dommages-intérêts à des personnes victimes de la destruction de leur environnement. L'étude du jugement est néanmoins intéressante à plusieurs niveaux. Premièrement, elle démontre l'importance que revêt, afin d'évaluer la suffisance de la persécution, l'appréciation de la relation qu'entretient la personne qui fuit un État avec l'environnement qu'elle quitte. Les victimes abandonnent un environnement qu'elles estiment être devenu inhabitable. L'affaire illustre donc quels types d'informations doivent être considérées dans l'évaluation du volet subjectif de la persécution. Deuxièmement, les défendeurs soulèvent l'incompétence du tribunal, au motif que les demandeurs auraient dû intenter leur action dans le pays qu'ils ont quitté. Les demandeurs doivent expliquer qu'ils ne pouvaient procéder ainsi, à cause d'une crainte objective d'être persécutés. Le jugement mentionne, par conséquent, la nature des éléments qui doivent être pris en compte dans l'appréciation du volet objectif de la persécution. Troisièmement, les personnes fuient à cause de la crainte d'être persécutées pour le motif de leur appartenance au groupe racial des noirs et au groupe social des démunis. Ce troisième point sera abordé dans la deuxième partie $(\mathrm{A})(\mathrm{b})$.

Il est important de rappeler les faits de l'affaire Sarei v. Rio Tinto (i), avant de se pencher sur les deux premiers éléments mentionnés dans le paragraphe précédent. Cette décision permet, en effet, d'illustrer le contenu des critères subjectifs et objectifs dans une situation de dégradation de l'environnement (ii).

\section{i) Les faits de l'affaire Sarei v. Rio Tinto}

La compagnie Rio Tinto opère, depuis 1972, l'une des plus importantes mines de cuivre du monde sur l'île de Bougainville en Papouasie Nouvelle-Guinée. L'aménagement de la mine nécessite le déménagement de villages et la destruction d'une superficie importante de la forêt tropicale de l'île. La compagnie accepte de verser une redevance de $19.1 \%$ des profits de la mine au gouvernement, afin qu'il collabore au projet. Ces profits constituent une partie importante des revenus de l'État ${ }^{44}$. Dès le creusage de la mine, dans les années 60 , des tensions surgissent entre la population et la

44. Ces redevances représentaient $80 \%$ des revenus de l'État au milieu des années 80; C. M. Kozoll, ibid. à la p. 304. 
compagnie, tout d'abord sur le montant des indemnités versées et par la suite, sur les activités de la compagnie.

Les opérations minières apportent leur lot de malheurs environnementaux. La contamination des cours d'eau a comme conséquence la destruction des ressources halieutiques, causant des pertes d'emploi chez les pêcheurs et tous les problèmes sociaux qui en découlent, notamment une modification du régime alimentaire des résidants de l'île ${ }^{45}$. La pollution atmosphérique occasionne des maladies respiratoires parmi la population et transforme le climat de l'île. Le changement climatique dévaste les cultures et amène des pertes d'emploi, cette fois-ci chez les agriculteurs. L'accumulation de déchets industriels sur les sols détruit une grande partie de la forêt tropicale, ce qui engendre des mises à pied dans le secteur forestier.

Des malaises sociaux importants naissent des opérations de la compagnie : alcoolisme, anxiété, tensions sociales et violence, qui dégénèrent en une guerre civile en 1988. Les événements amènent la fermeture de la mine et la compagnie menace le gouvernement de retirer tous ses investissements de l'île, si ce dernier ne réprime pas les émeutes avec fermeté. Le gouvernement décrète un embargo de l'île, auquel collabore la compagnie. Le blocus empêche la distribution de médicaments à la population, ce qui cause la mort de 200 enfants lors des deux premières années de son instauration. Par la suite, des milliers de Bougainvilliers décèdent ou quittent l'île.

ii) Le contenu des critères subjectifs et objectifs dans une situation de dégradation de l'environnement, à la lumière de l'affaire Sarei v. Rio Tinto $^{46}$

La preuve démontre dans l'affaire Sarei v. Rio Tinto que les activités minières et la dégradation consécutive de l'environnement détruisit les pêcheries, l'agriculture et la foresterie. Ce sont donc les assises socio-

45. Des problèmes d'obésité importants chez les femmes et les enfants qui abandonnèrent leurs habitudes alimentaires traditionnelles au profit de plats occidentaux surgelés, ibid. à la p. 303.

46. Les commentaires formulés dans cette sous-section sont inspirés de l'analyse réalisée par C. M. Kozoll, ibid. 
À la recherche d'un statut juridique pour
(2006) 37 R.D.U.S. $\quad \begin{gathered}\text { les migrants environnementaux transfrontaliers : } \\ \text { la problématique de la notion de réfugié }\end{gathered}$

économiques de l'île de Bougainville, reposant sur l'exploitation des ressources premières du milieu, qui sont anéanties. Les Bougainvilliers considèrent que le lien symbiotique qu'ils entretiennent avec l'environnement, qui constitue une caractéristique fondamentale de leur mode de vie autochtone, est rompu. La crainte ressentie par les personnes qui fuient l'île est suscitée par les trois circonstances suivantes. La première concerne la destruction du milieu, duquel les Bougainvilliers tirent presque intégralement leurs moyens de subsistance. Ce facteur à lui seul menace la survie de la population. La deuxième a trait aux violences exercées par les forces de l'ordre à l'égard des contestataires, lors des émeutes. Les témoignages indiquent, en effet, que des manifestations populaires à l'encontre des activités de la compagnie furent réprimées brutalement par les forces militaires. La troisième touche les décès observés dans la population, suite à l'embargo commercial qui empêche la distribution de médicaments.

Le dénominateur commun de ces situations réside dans la menace qu'elles imposent à l'existence même des personnes. Les autochtones de l'île manifestent un état d'esprit qui est inspiré par la crainte d'une atteinte à leur intégrité physique et psychique, menaçant directement leur droit à la vie. Les personnes qui fuient l'île rencontrent ainsi le critère subjectif de la persécution.

Quant au critère objectif, le tribunal conclut que les résidants se fondent sur une crainte légitime, c'est-à-dire supportée par les faits mis en preuve, qu'ils ne pouvaient intenter leur action en Papouasie NouvelleGuinée, à cause du contexte politique y prévalant. Le rôle joué par le gouvernement afin de faciliter l'établissement de la compagnie et le soutien qu'il lui apporta par la suite, en contrepartie du versement d'une partie des profits qu'elle réalisait, témoignent des liens étroits qu'entretenaient les deux entités. Les importantes redevances versées par la compagnie à l'État rendent légitime la crainte des personnes à l'égard d'un gouvernement complice d'opérations destructrices de l'environnement.

Le gouvernement se comportait comme l'agent actif d'une personne morale dans la répression des émeutes engendrées par la situation du pays. Il perdait ainsi toute légitimité afin d'agir comme un arbitre impartial affecté à la protection des intérêts des nationaux. Les demandeurs auraient craint pour 
leur sécurité s'ils avaient intenté le recours en Papouasie Nouvelle-Guinée. Le tribunal conclut ainsi, que c'est «avec raison» qu'ils ne pouvaient retourner sur l'île afin d'intenter une poursuite contre la compagnie.

Des autorités compétentes appelées à déterminer si les personnes qui ont fui la Papouasie Nouvelle-Guinée rencontraient les critères d'un réfugié au sens de la Convention de 1951 et du Protocole de 1967, en seraient, presque assurément, arrivées à la même conclusion que le tribunal saisi de l'affaire Sarei v. Rio Tinto, quant à l'évaluation du contexte social prévalant dans le pays. Le tribunal indique, en effet, que la crainte légitime des résidents est appuyée par une situation factuelle claire.

\section{b) Les limites à considérer la dégradation de l'environnement comme une forme de persécution}

Le Guide mentionne expressément la famine et les catastrophes naturelles, comme des exemples de causes qui ne seraient pas valables afin de qualifier une personne de réfugiée ${ }^{47}$. Le Guide laisse donc supposer, à première vue, que prise isolément, la crainte d'une menace à la vie suscitée par une perturbation importante de l'environnement, ne pourrait pas créer un état d'esprit qui éveillerait un sentiment de persécution chez un individu ${ }^{48}$.

Le Guide indique, cependant, que les causes de migrations des personnes, autres que la crainte d'être persécuté pour l'un des motifs mentionnés à la Convention de 1951 et au Protocole de 1967, parmi lesquelles se retrouvent les catastrophes naturelles :

(...) peuvent ne pas demeurer tout à fait étrangères au processus de détermination de la qualité de réfugié, car il convient de tenir compte de toutes les circonstances pour se faire une idée exacte de la situation de celui qui demande le statut de réfugié ${ }^{49}$.

47. $\quad$ Guide, supra note 31, art. 39.

48. Le guide indique, cependant, que les victimes de la famine et des catastrophes naturelles ne seraient pas considérées comme des réfugiés, «à moins que de surcrô̂t ces victimes ne craignent avec raison d'être persécutées pour l'un des motifs prévus», ibid., art. 39. 
À la recherche d'un statut juridique pour
(2006) 37 R.D.U.S. $\quad$ les migrants environnementaux transfrontaliers :
la problématique de la notion de réfugié

Cela signifie qu'une personne ne pourrait espérer recevoir l'asile dans un pays, en se faisant reconnaître le statut de réfugié, sur le seul fondement de la survenance d'une catastrophe naturelle. Cet élément conforterait, toutefois, la légitimité d'une demande appuyée sur l'un des motifs prévus à la Convention de 1951 et au Protocole de 1967.

La persécution est le résultat d'un acte gouvernemental à l'égard des individus. Comme le Guide l'indique: «on entend normalement par persécution une action qui est le fait des autorités d'un pays» ${ }^{50}$. Certains auteurs $^{51}$ considèrent sur cette base que des désastres environnementaux peuvent constituer une forme de persécution, en eux seuls, lorsqu'ils résultent de l'action ou de l'inaction des gouvernements. Une modification du milieu qui aurait une origine naturelle n'ouvrirait pas la porte, selon eux, à une demande pour obtenir le statut de réfugié, tandis qu'une modification du milieu, consécutive à une action ou une omission de l'homme, le ferait. Cette position est conforme avec la définition du mot «persécution»: «Mauvais traitement dont on est la victime. Maltraitance.» ${ }^{52}$.

Il peut donc être déduit, selon la thèse soutenue, que la dégradation de l'environnement constituerait une forme de persécution supportant une demande pour l'obtention du statut de réfugié, dans la seule hypothèse où elle résulte des décisions ou omissions de l'État. Il est facilement démontrable que les gouvernements jouent un rôle important dans la genèse de nombreux désastres environnementaux. Ils s'abstiennent d'agir dans certaines circonstances, ou retardent indûment l'adoption de mesures dans d'autres. Cette attitude est évidente en ce qui a trait aux changements climatiques. Dans certains cas, ils prennent carrément de mauvaises décisions. Dans toutes ces situations, les dirigeants accentuent les risques de dommages à la population, en la rendant plus vulnérable aux modifications de l'environnement. Les migrants environnementaux seraient alors les victimes d'une persécution gouvernementale ${ }^{53}$.

\footnotetext{
50. Ibid., art. 65 .

51. Voir notamment J. B. Cooper, supra note 5 à la p. 502; Leon Gordenker, «Refugees» International Politics $\mathrm{n}^{\mathrm{O}} 30$ (1987).

52. Le Petit Robert, 2002, Elle ne pourrait cependant s'appuyer sur cette autre définition donnée par le dictionnaire «Traitement injuste et cruel infligé avec acharnement».

53. J. B. Cooper, supra note 5 à la p. 502; G. S. McCue, supra note 16 aux pp. 170-73.
} 
Dans l'Affaire Sarei v. Rio Tinto, le gouvernement a, par son action, soutenu l'entreprise Rio Tinto. C'est pour cette raison que les Bougainvilliers le tiennent tout aussi responsable que la compagnie, du désastre environnemental survenu dans l'île et qui a rendu le milieu inhabitable.

Bien qu'un lieu inhospitalier constitue un motif important afin de quitter une région lorsque le milieu se dégrade, il peut être plus aisément prouvable, dans le cas où l'environnement rend une région carrément inhabitable, que la cause fondamentale du déplacement des personnes réside dans les actions et omissions du gouvernement. Une modification mineure de l'environnement se distinguerait du désastre environnemental, justement parce que ce dernier comporte nécessairement le déplacement d'une population. Lorsqu'une population entière doit quitter un environnement où il est impossible de survivre, dû aux effets induits par le réchauffement planétaire par exemple, ses membres constituent des réfugiés environnementaux qui fuient une persécution causée par leurs gouvernements $^{54}$. Le fait que le phénomène résulte de l'inaction des gouvernements collectivement n'altère pas la responsabilité de chacun de ceux-ci ${ }^{55}$.

Cette théorie laisse cependant entière la question de la détermination de la part de la responsabilité des gouvernements dans la survenance des catastrophes environnementales qui rendent une région inhabitable. Comment peut-il être déterminé qu'une modification de l'environnement est issue de causes purement naturelles ou qu'elle résulte de l'intervention ou de l'inaction des dirigeants d'un État? Les ouragans Katrina et Rita qui ont dévasté la Louisiane et le Texas en septembre 2005 constituent-ils des catastrophes purement naturelles ou résultent-elles de l'inaction du gouvernement états-unien à lutter contre les changements climatiques? Bien que les scientifiques indiquent que les études réalisées sur le réchauffement planétaire ne permettent pas d'établir une corrélation directe entre l'effet de serre et la force des ouragans, ils s'accordent néanmoins pour affirmer qu'il est fortement probable qu'un lien de cause à effet existe entre les événements.

54. Cooper cite notamment la désertification et l'accident industriel de Tchernobyl afin d'étayer sa thèse de la responsabilité des gouvernements; J. B. Cooper, ibid. à la p. 503 et $\mathrm{s}$.

55. J. B. Cooper, ibid. à la note 185. 
À la recherche d'un statut juridique pour
(2006) 37 R.D.U.S. $\quad$ les migrants environnementaux transfrontalier

la problématique de la notion de réfugié

Une solution consisterait à évaluer le comportement des États, dans de telles circonstances, sur la base du principe de précaution ${ }^{56}$ formulé comme suit dans la Déclaration de Rio sur l'environnement et le développement :

Pour protéger l'environnement, des mesures de précaution doivent être largement appliquées par les États selon leurs capacités. En cas de risque de dommages graves ou irréversibles, l'absence de certitude scientifique absolue ne doit pas servir de prétexte pour remettre à plus tard l'adoption de mesures effectives visant à prévenir la dégradation de l'environnement ${ }^{57}$.

Ainsi, lorsqu'il semble raisonnablement établi d'un point de vue scientifique, que des désastres environnementaux auraient pu être significativement mitigés, si les gouvernements avaient pris des mesures appropriées en temps et lieux opportuns, ces derniers devraient alors assumer leur responsabilité dans le cas des migrations de populations qui en résultent. Le gouvernement états-unien est responsable, pour une partie non négligeable, des déplacements de population liés aux ouragans Katrina et Rita, non seulement parce qu'il a toujours entretenu de sérieuses réserves quant au réchauffement du climat, mais aussi parce qu'il contribue grandement à ce dernier. Il est ainsi aisé de comprendre son retrait du Protocole de Kyoto. L'un des éléments à prendre en compte, afin d'attribuer une part de responsabilité à un État dans la survenance d'un désastre

56. Sur le principe de précaution, consulter: Pierre-François Mercure et John Edwin Sullivan, «Le principe de précaution en droit canadien : une intégration tributaire de l'ordre économique international», Revue roumaine de droit de l'environnement, An III, nr. 2(6)/2005 à la p. 5; Alexandre Kiss et Jean-Pierre Beurier, Droit international de l'environnement, $3^{\mathrm{e}}$ éd., Paris, Pedone, 2004 aux pp. 136-38; Hélène Trudeau, «Du droit international au droit interne: l'émergence du principe de précaution en droit de l'environnement» (2003) 28 Queen's L.J. 455; Cass R. Sunstein, «Beyond the Precautionary Principle» (2003) 151 U. Pa. L. Rev. 1003; Maurice Arbour, «Le principe de précaution dans le contexte du commerce international: une intégration difficile» (2002) 43 C. de D. à la p. 5; J. Tickner, C. Raffensperger et N. Myers, The Precautionary Principle in action, A handbook, first edition written for the Science and Environmental Health Networks. En ligne : http://biotech-info.net/handbook.pdf (date d'accès 28 septembre 2006); Nicolas de Sadeleer, Les principes du pollueur-payeur, de prévention et de précaution. Essai sur la Genèse et la Portée juridique de quelques principes du droit de l'Environnement. Paris, Bruylant, AUF, 1999; Philippe Kourilsky et Geneviève Viney, Le Principe de Précaution: rapport au premier ministre, Paris, Odile Jacob, 2000.

57. Déclaration de Rio sur l'environnement et le développement, (13 juin 1992), Doc. N.U. A/Conf. 151/5/Rev.1. Principe 15. 
À la recherche d'un statut juridique pour

les migrants environnementaux transfrontaliers : (2006) 37 R.D.U.S.

la problématique de la notion de réfugié

environnemental, serait assurément de considérer que des personnes sont obligées de se déplacer afin de ne pas subir les inconvénients qui en résultent.

Si les désastres environnementaux peuvent constituer une forme de persécution lorsqu'ils sont attribuables au rôle de l'État, peut-il être soutenu qu'elle découle de l'un des motifs prévus à la Convention de 1951 et au Protocole de 1967 : la race, la religion, la nationalité, l'appartenance à un certain groupe social ou les opinions politiques? Tel que mentionné, la deuxième partie $(\mathrm{A}) \mathrm{b}$ ) tente de répondre à cette question. Cependant, cette dernière ne peut être abordée que dans le cadre de la définition d'un cadre normatif international portant sur les migrants environnementaux.

\section{Deuxième partie : La définition d'un cadre normatif international}

Deux avenues s'offrent à la communauté internationale afin d'adapter le contexte juridique à la problématique des migrants environnementaux. La première consiste à amender les conventions en vigueur $(\mathrm{A})$ et la deuxième vise à adopter une convention portant sur les réfugiés environnementaux $(\mathrm{B})$.

\section{A) Les amendements au droit positif des réfugiés}

L'octroi d'un statut juridique aux migrants environnementaux pourrait résulter de la reconnaissance du droit à la qualité de l'environnement dans la Convention de 1951 et le Protocole de 1967 (a). Il est aussi, alternativement, susceptible d'être conféré, en ajoutant la notion de pauvreté aux motifs de la persécution, dans un contexte de modification de l'environnement (b).

\section{a) La reconnaissance du droit à la qualité de l'environnement}

La Convention de 1951 permet d'articuler le droit de chercher asile, que codifie la Déclaration universelle des droits de l'homme ${ }^{58}$. La Convention de 1951 reconnaît expressément que le statut de réfugié résulte d'un déni de respecter certains droits humains, prévus par la Charte des

58. «Devant la persécution, toute personne a le droit de chercher asile et de bénéficier de l'asile en d'autres pays»: Déclaration universelle des droits de l'homme, 1948, Doc. N.U. A/810, p. 71, art. 14(1). 
Nations Unies $^{59}$ et la Déclaration universelle des droits de l'homme ${ }^{60}$. Les droits humains reconnus par la Convention de 1951 et qui ne peuvent faire l'objet d'une persécution peuvent être classés dans deux catégories : les droits protégés directement et ceux qui le sont indirectement. La première regrouperait les cinq droits prévus dans la Déclaration universelle des droits de l'homme: le droit à la non-discrimination fondée sur la race ou la nationalité, la liberté de religion, de pensée et d'appartenance à un groupe. La deuxième rassemblerait des droits liés aux premiers et des droits qui permettent leur mise en œuvre ${ }^{61}$. Le droit à la vie serait évidemment protégé, puisqu'une menace à la vie peut mener au décès, forme ultime de la persécution.

Certains auteurs proposent d'élargir la définition du terme réfugié et, pour y arriver, d'étendre le spectre des droits protégés par la Convention de 1951 afin de tenir compte de du développement des droits de la personne ${ }^{62}$, qui a amené la reconnaissance du droit à la qualité de l'environnement. L'évolution s'est réalisée en deux étapes; dans un premier temps en 1947, lors de l'adoption de la Déclaration universelle des droits de l'homme et dans un second, en 1966, lors de l'adoption du Pacte international relatif aux droits civils et politiques ${ }^{63}$ et du Pacte international relatif aux droits économiques, sociaux et culturels ${ }^{64}$.

L'article 3 de la Déclaration universelle des droits de l'homme proclame : «tout individu a droit à la vie, à la liberté et à la sûreté de sa personne». L'article 22, quant à lui, énonce :

59. $\quad$ Charte de l'organisation des Nations Unies, 1 R.T.N.U. xvi, R.T.C. 1945 no. 7.

60. Préambule de la Convention de 1951, supra note 11: Considérant que la Charte de Nations Unies et la Déclaration universelle des droits de l'homme (...) ont affirmé le principe que les êtres humains, sans discrimination, doivent jouir des droits de l'homme et des libertés fondamentales.

61. Par exemple, le droit de se constituer en syndicats, prévu à l'article 22 du Pacte international relatif aux droits civils et politiques, serait lié au droit à la liberté de réunion et d'association prévu à l'article 20 de la Déclaration universelle des droits de l'homme, supra, note 58. Pacte international relatif aux droits civils et politiques, 1966, 999 R.T.N.U. 107; R.T.C. 1976, no. 47.

62. J. B. Cooper, supra note 5 aux pp. 490-94; D. Keane, supra note 1 à la p. 215.

63. Pacte international relatif aux droits civils et politiques, supra note 61.

64. Pacte international relatif aux droits économiques, sociaux et culturels, 1966, 943 R.T.N.U. 13; R.T.C. 1976, nº 46. 
Toute personne, en tant que membre de la société, a droit à la sécurité sociale et elle est fondée à obtenir la satisfaction des droits économiques, sociaux et culturels indispensables à sa dignité et au libre développement de sa personnalité, grâce à l'effort national et à la coopération internationale, compte tenu de l'organisation et des ressources de chaque pays.

L'article 25 du même document indique : «Toute personne a droit à un niveau de vie suffisant pour assurer sa santé, son bien-être et ceux de sa famille, notamment pour l'alimentation, l'habillement, le logement, les soins médicaux ainsi que pour les services sociaux nécessaires. (...)». Les paragraphes 1(2) du Pacte international relatif aux droits civils et politiques et du Pacte international relatif aux droits économiques, sociaux et culturels mentionnent le droit inhérent des peuples de disposer librement de leurs ressources naturelles et de ne pas être privés de leurs propres moyens de subsistance ${ }^{65}$.

Ces dispositions reconnaissent le droit d'être à l'abri d'un contexte environnemental qui menace directement ou indirectement l'existence. Tel qu'il est libellé dans la Déclaration universelle des droits de l'homme et les Pactes, le droit d'être à l'abri d'une dégradation intolérable de l'environnement requiert une protection équivalente aux cinq droits protégés par la Convention de 1951 et le Protocole de 1967. Ce droit constitue la genèse du droit à la qualité de l'environnement codifié, en 1972, dans la Déclaration de Stockholm sur l'environnement ${ }^{66}$. La Convention de 1951 et le Protocole de 1967 devraient donc être modifiés afin de refléter l'évolution des droits de la personne qui reconnaît le droit à la qualité de l'environnement. Il devrait, alors, être procédé à l'élargissement de la portée de la Convention de 1951 et du Protocole de 1967 aux déplacements interétatiques, pour le motif d'une oppression gouvernementale affectant le droit des individus à un environnement sain.

65. Voir aussi l'art. 47 du Pacte international relatif aux droits civils et politiques au même effet, supra note 61.

66. Déclaration de Stockholm sur l'environnement, 1972, Doc. N.U.A./CONF. 48/14/Rev. 1, aux pp. 3-6. 
Selon Keane, une telle éventualité est improbable, car elle se heurterait à une farouche opposition de la part des États ${ }^{67}$. Selon l'auteur, les États considèrent qu'une modification de la définition de réfugié constituerait un risque important d'atteinte au principe de la souverainetét ${ }^{68}$, auquel ils sont fermement attachés. En effet, les migrations environnementales se produisent généralement dans un cadre infra-étatique et les États rejetteraient probablement toute proposition visant à leur imposer des normes internationales applicables aux migrations de populations se déroulant sur leur territoire.

Cette hypothèse buterait contre l'opposition des États développés pour deux autres raisons. Premièrement, ces derniers craignent un afflux de migrants à leurs portes ${ }^{69}$. Deuxièmement, ils s'inquiètent de voir leur contribution financière au HCNUR augmenter significativement. Les dirigeants de l'organisme partagent aussi cette préoccupation. Ils estiment que les maigres ressources dont ils disposent actuellement seraient insuffisantes afin de financer une augmentation significative des réfugiés environnementaux.

Seul un amendement mineur à la définition des articles 1 de la Convention de 1951 et du Protocole de 1967 serait envisageable. Ce dernier ne pourrait tenir compte que de la persécution exercée sur des personnes qui ont fui un État qui nie leur droit à un environnement sain et ne pourrait viser les migrations internes. Il serait alors requis de modifier comme suit la définition du terme réfugié : toute personne (...) craignant avec raison d'être persécutée du fait (...) d'une modification de l'environnement, en laissant intacte la suite de la définition : «(..) qui (...) se trouve hors du pays dont elle a la nationalité et qui ne peut ou, du fait de cette crainte, ne veut se réclamer de la protection de ce pays $(\ldots){ }^{70}$.

67. D. Keane, supra note 1 aux pp. 215-16.

68. Consacré par l'art. 2(7) de la Charte de l'Organisation des Nations Unies, supra note 59. Ibid.

69. J. B. Cooper, supra note 5 à la p. 500; G. S. McCue, supra note 16 à la p. 177. Les études tendent toutes à démontrer que les migrations environnementales augmenteront significativement au cours des prochaines décennies et que les destinations privilégiées seront les pays développés. G. S. McCue, ibid. à la p. 188.

70. Supra note 33. 
À la recherche d'un statut juridique pour

les migrants environnementaux transfrontaliers : $\quad$ (2006) 37 R.D.U.S.

la problématique de la notion de réfugié

L'attribution d'un statut légal aux migrants environnementaux pourrait aussi résulter de l'ajout de la notion de pauvreté aux motifs de persécution.

\section{b) La pauvreté comme motif de persécution, dans un contexte de modification de l'environnement}

Comme il sera démontré, parmi les cinq motifs de persécution, seule l'appartenance à un certain groupe social présente un certain intérêt pour déterminer si le statut de réfugié peut être conféré à des migrants environnementaux. Il pourrait leur être attribué, puisqu'ils appartiendraient au groupe social des démunis (ii). Il est cependant requis, préalablement, d'élaborer sur la difficulté d'attribuer à la race un motif de persécution environnementale (i).

\section{i) La difficulté d'attribuer à la race un motif de persécution environnementale}

À la suite du passage des ouragans Katrina et Rita, il a été avancé que le gouvernement fédéral américain avait déployé des secours avec moins d'empressement dans l'État de la Louisiane, constitué d'une majorité de citoyens de race noire, que si les catastrophes s'étaient produites dans une ville à majorité blanche du nord des États-Unis ${ }^{71}$.

Si des Néo-Orléanais de race noire avaient demandé l'asile politique au Mexique ou au Canada ${ }^{72}$ suite aux ouragans, auraient-ils pu invoquer la persécution fondée sur la race afin d'appuyer leur demande? Si la question est posée, elle restera sans doute sans réponse dans l'éventualité où aucune demande de statut de réfugié n'est déposée auprès des gouvernements de ces États. Ces événements laissent cependant entrevoir qu'il n'est pas exclu que

71. John Kenneth Galbraith, «L'art d'ignorer les pauvres», Le Monde Diplomatique (octobre 2005) à la p. 6.

72. Il est à noter que le Canada a adopté des mesures visant à faciliter l'immigration des victimes du Tsunami survenu le 26 décembre 2004. Le 7 juin 2005, Citoyenneté et Immigration Canada avait délivré 366 visas de résident permanent et accordé le statut de résident à 278 personnes. Communiqué de Citoyenneté et Immigration Canada, (5 décembre 2005) en ligne : <http://www.cic.gc.ca/francais/nouvelles/05/0516\%2Df.html>. 
À la recherche d'un statut juridique pour
(2006) 37 R.D.U.S. $\quad \begin{gathered}\text { les migrants environnementaux transfrontaliers : } \\ \text { la problématique de la notion de réfugié }\end{gathered}$

la persécution fondée sur la race puisse constituer un motif à l'appui d'une demande de statut de réfugié, suite à des catastrophes environnementales.

C'est d'ailleurs ce que révèle l'Affaire Sarei v. Rio Tinto. Il a été, en effet, démontré que les Bougainvilliers, qui sont des personnes de race noire, subissaient une discrimination à divers degrés de la part de l'entreprise. Cette dernière privilégia l'embauche de travailleurs de race blanche venus de l'extérieur. Ainsi, la majorité des travailleurs de la mine était constituée de travailleurs étrangers. Les conditions de travail des autochtones embauchés étaient nettement inférieures à celles des blancs. Tel que rapporté dans le jugement :

Rio Tinto viewed the people of Bougainville as inferior due to their color and culture and, therefore, intentionnally violated their rights ${ }^{73}$ (...).

Discrimination was a motivating factor in Rio's actions in Bougainville and around the world. In South Africa, Rio paid a black migrant labor force an average wage below the minimum set by the South African Institute of Race Relations. At another mine, mine laborers were housed in «appalling temporary camps» in slave-like conditions. Furthermore, Rio has not hesitated to displace indigenous communities to implement various projects, forcing the relocation of villages and the extinction of culture ${ }^{74}(\ldots)$.

Les chances de réussite d'une demande de statut de réfugié, suite à des catastrophes environnementales, fondée sur la persécution dont le motif est la race, si elles existent, sont néanmoins incertaines. Il n'existe aucun précédent à ce sujet. L'Affaire Sarei v. Rio Tinto n'ayant pas eu à se prononcer sur l'octroi du statut de réfugié, tel que mentionné à la première partie (B) a).

S'il est difficile d'envisager que les victimes des ouragans Katrina et Rita puissent réussir à faire la preuve que l'inaction des gouvernements fédéral, étatique et municipal américains dans le rehaussement des digues protégeant la Nouvelle-Orléans constituait un motif de persécution de la

73. Sarei v. Rio Tinto, supra note 43 à la p. 1124; C. M. Kozoll, supra note 42 à la p. 306.

74. Sarei v. Rio Tinto, ibid, à la p. 50; C. M. Kozoll, ibid. à la p 301. 
population noire de la ville, il semble plus réaliste d'envisager de prouver que cette inaction affectait prioritairement les pauvres de la ville.

\section{ii) L'appartenance au groupe social des démunis}

Une réflexion sur ce sujet soulève la question de l'identification du profil socio-économique des migrants environnementaux. Un recensement des catastrophes environnementales révèle que ces dernières se produisent, en grande partie, dans des pays en développement ou dans des pays en transition. Elles frappent donc prioritairement les personnes les plus défavorisées de la planète.

Les modifications lentes de l'environnement, notamment le réchauffement planétaire, et toutes les conséquences qui en découlent, soit : inondations, pénuries d'eau potable, désertification, famines, ouragans tropicaux, etc., affecteront principalement les pays en développement les plus pauvres, ceux compris dans la catégorie des pays les moins avancés ${ }^{75}$, parmi lesquels sont recensés la presque totalité des micro-États de l'océan pacifique, mais aussi de nombreux États victimes de la désertification ${ }^{76}$. Ce type d'altération de l'environnement n'épargnera cependant pas les pays développés. Les effets de la hausse du niveau des océans due au réchauffement de la planète, affecteront indéniablement les Pays-Bas où une partie importante du territoire de l'État est située sous le niveau de la mer ${ }^{77}$.

75. Au nombre de 50 en 2005. Ils sont aussi nommés «pays les moins développés». Programme des Nations unies sur le développement (PNUD), «Rapport mondial sur le développement humain» (2005), en ligne : <http://hdr.undp.org/reports/global/ 2005/francais/pdf/HDR05_fr_backmatter.pdf>.

76. Sur la désertification, voir aussi Michelle Leighton, «Desertification and Migration» dans Johnson, Pierre Marc, Karol Mayrand et Marc Paquin (dir.) Governing Global Desertification, Linking Environmental Degradation, Poverty and Participation, Londres, Ashgate Press [à paraître en 2006]; Sommet planète Terre+5. Convention de Nations Unies sur la lutte contre la désertification: nouvelle réponse à un problème séculaire, Département de l'information de l'ONU, DPI/1870/SD aux pp. 49-60 (mars 1997).

77. La réduction des émissions de CO2 constitue, depuis 1995, l'élément central de la stratégie environnementale du pays. Consulter, à ce sujet en ligne : <http://www.minbuza.nl/default.asp?CMS_ITEM=CF683C976E364285BF7E4660CE72 0DF9X3X46518X23> (5 janvier 2006). 
Il reste cependant que, même lorsqu'une catastrophe environnementale frappe un pays développé, les couches défavorisées de la population subissent plus durement que les autres les conséquences de la modification du milieu et constituent la proportion la plus importante des personnes obligées de fuir pour survivre. Cette situation découle du fait que les plus démunis ne disposent pas des ressources financières afin d'acquérir des biens qui leur permettent de faire face aux catastrophes environnementales en toute sécurité. Les ouragans Katrina et Rita ont, en effet, démontré que les victimes les plus touchées par leur action dévastatrice étaient les citoyens les plus pauvres de la Louisiane, car ils habitaient dans des zones sensibles aux inondations, mais aussi dans des habitations plus vulnérables aux forces naturelles libérées par ces cataclysmes.

C'est en se fondant sur l' «appartenance à un certain groupe social» au sens de l'art. 1) a) 2) de la Convention de 1951 et de l'art. 1) 2) du Protocole de 1967, que l'auteur Cooper suggère que les victimes de catastrophes environnementales pourraient être considérées comme des réfugiés ${ }^{78}$. Cette mention subsumerait les autres: race, religion, nationalité, opinion politique. Cooper indique qu'elle agirait comme suit : «It acts as a «catch-all» for individuals not falling into the remaining categories» ${ }^{79}$.

La catégorie aurait été ajoutée afin d'inclure les personnes persécutées pour des raisons autres que celles spécifiquement indiquées ${ }^{80}$. Il ne fait aucun doute que les personnes économiquement défavorisées, qui constituent la grande majorité des résidants des pays en développement et qui seraient victimes de catastrophes écologiques, pourraient être incluses dans cette catégorie. Cette affirmation trouverait tout son sens lors de migrations consécutives aux changements climatiques. Ces réfugiés se caractériseraient par leur appartenance à des groupes sociaux issus d'États incapables, politiquement, de forcer les pays développés à réduire leurs émissions de gaz à effet de serre. Il peut être affirmé :

It is precisely because the citizens of the nations to be most severely affected by global warming are members of «social group» without

78. J.B. Cooper, supra note 5 à la p. 521.

79. Ibid. à la p. 521.

80. Ibid. à la p. 522. 
political power to protect their environment that the governements of the developed world continue to expose them to the risks of environmental degradation ${ }^{81}$.

Les personnes défavorisées économiquement, mais cette fois-ci issues de pays développés, pourraient aussi être persécutées en raison de leur appartenance au groupe social des démunis. Le Guide indique d'ailleurs :

par groupe social on entend normalement des personnes appartenant à un groupe ayant (...) le même statut social. (...) L'appartenance à un certain groupe social peut être à l'origine de persécutions parce que (...) l'activité économique de ses membres, voire l'existence même du groupe social en tant que tel, sont considérées comme un ${ }_{\text {obstacle à la mise en oeuvre des politiques gouvernementales }}^{82}$ (nos soulignements).

Dans le contexte néo-libéral qui imprègne les politiques publiques des États depuis la fin de la Seconde Guerre mondiale, les démunis, qui partagent le même statut social, constituent «un obstacle à la mise en œuvre des politiques gouvernementales» de droite.

Le mouvement écologiste, né dans les années 60, repose sur une idéologie fondée sur la justice sociale, dont la protection de l'environnement constitue le pivot ${ }^{83}$. Certaines organisations environnementales, non gouvernementales, issues de la société civile dans les années 70, principalement aux États-Unis, se sont données la mission de représenter les intérêts de segments défavorisés de la population ${ }^{84}$. Elles considèrent que ceux-ci souffriraient d'un déficit démocratique afin de promouvoir leur droit à un environnement sain. Ces organisations revendiquent, par conséquent, d'agir au nom de personnes appartenant au groupe social des démunis. Les migrants environnementaux pourraient ainsi obtenir le statut de réfugié en se réclamant d'appartenir aux groupes sociaux ciblés par ces organismes.

81. Ibid. à la p. 525.

82. Guide, supra note 31, art. 77-78.

83. J. B. Cooper, supra note 5, à la note 237.

84. Notamment le groupe The Environmental Justice Movement, J.B. Cooper, ibid. aux pp. 523-24. 
Il apparaît évident dans l'affaire Sarei v. Rio Tinto, qu'une demande de statut de réfugié aurait eu de bonnes chances d'être accordée, même en l'absence du fondement juridique de l'existence d'une guerre civile sur l'île de Bougainville ${ }^{85}$. Les conditions pour qu'il en eût été ainsi sont toutefois assez restrictives. La personne aurait dû démontrer que les dommages environnementaux subis étaient suffisamment importants pour qu'ils menacent le mode de vie, au sens large, des résidants, c'est-à-dire leurs moyens de subsistance, leur culture, etc., que le gouvernement local a participé, ou, à tout le moins a acquiescé à leur commission et qu'ils ont affecté des démunis. Bien que la responsabilité de conférer à une personne le statut de réfugié relève des États, il revient à leurs tribunaux d'interpréter ce terme à la lumière de la Convention de 1951 et du Protocole de 1967. Il serait alors souhaitable, afin d'éviter toute ambiguïté sur le sens de l'expression «appartenance à un certain groupe social» ${ }^{86}$, de modifier la définition de réfugié comme suit: toute personne (...) craignant avec raison d'être persécutée du fait de son appartenance à un certain groupe social, notamment celui des victimes de la dégradation de l'environnement (...).

Ces commentaires amènent à considérer l'avenue de la rédaction d'une convention adaptée aux migrations environnementales, hors du contexte propre à la notion de réfugié, telle que comprise dans la Convention de 1951 et le Protocole de 1967.

\section{B) L'adoption d'une convention portant sur les migrations environnementales}

L'adoption d'une convention implique de concilier ses objectifs avec les particularismes régionaux (a). Sans énumérer toutes les dispositions d'une

85. Les réfugiés de guerre, qu'il s'agisse de conflits armés nationaux ou internationaux «ne sont pas normalement considérés comme des réfugiés au regard de la Convention de 1951 et du Protocole de 1967. Ils jouissent, cependant, de la protection prévue par d'autres instruments internationaux, tels que la Convention de Genève de 1949 sur la protection des victimes de guerre et le Protocole additionnel de 1977 aux Conventions de Genève de 1949 relatif à la protection des victimes de conflits armés internationaux». Guide, supra note 31, art. 164. La guerre civile ne constitue pas en soi un motif suffisant de crainte d'être persécuté. Guide, ibid., art. 98.

86. Convention de 1951, supra note 10, art 1) a) 2) et Protocole de 1967, supra note 11, art.

1) 2). 
À la recherche d'un statut juridique pour

les migrants environnementaux transfrontaliers: (2006) 37 R.D.U.S.

la problématique de la notion de réfugié

telle convention, il est utile d'exposer les principes directeurs qui l'animeraient (b).

\section{a) La conciliation des objectifs de la convention avec les particularismes régionaux}

Une convention portant sur les migrations environnementales devrait s'articuler autour de deux axes. En ce qui concerne le premier, elle aurait une portée horizontale, en ce qu'elle devrait prévoir des prescriptions différentes pour les migrations infra-étatiques et inter-étatiques. Les États auraient évidemment la possibilité de formuler une réserve quant à l'application des dispositions relatives aux migrations internes de populations, dans l'éventualité où certains d'entre eux considéreraient que ces dernières relèvent de leur domaine réservé ${ }^{87}$. Le deuxième axe conférerait une portée verticale à la convention. Elle devrait prévoir des modalités spécifiques applicables, d'une part, aux catastrophes environnementales et d'autre part, aux modifications lentes de l'environnement. Les migrations internes seraient traitées différemment selon qu'elles résultent de l'une ou l'autre des situations.

Quelques commentaires s'imposent relativement aux migrations inter-étatiques. Les personnes déplacées ont de plus grandes chances de pouvoir retourner à leur lieu d'origine dans le cas de catastrophes environnementales, que dans celui de modifications lentes de l'environnement. Il est, par conséquent, moins important de prévoir, dans la première situation, des mécanismes d'établissement et des mesures d'intégration permanentes des personnes déplacées dans l'État d'accueil, comme par exemple un accès facilité à la nationalité ou à la propriété foncière. Les dispositions applicables de la convention devraient, dans ce cas, avoir comme objectif prioritaire de porter une assistance aux migrants environnementaux à l'endroit où se produit la catastrophe. De telles mesures faciliteraient l'adhésion des pays développés à la convention, puisqu'elles

87. Consacré par l'article 2 (7) de la Charte des Nations Unies. Voir à ce sujet : G. ArangioRuiz, «Le domaine réservé : l'organisation internationale et le rapport entre le droit international et le droit interne» (1990) 225 R.C.A.D.I. 9. 
À la recherche d'un statut juridique pour
(2006) 37 R.D.U.S. $\quad$ les migrants environnementaux transfrontaliers :
la problématique de la notion de réfugié

tiendraient compte de leurs préoccupations, tel que mentionné précédemment $^{88}$.

En ce qui concerne la deuxième situation, celle des modifications lentes de l'environnement, des modalités spécifiques devraient être prévues. Lorsque les personnes quittent leur domicile au moment où les perturbations $\mathrm{du}$ milieu sont suffisamment importantes pour le rendre inhabitable, c'est généralement de façon indéfinie, sans possibilité réelle de retour. La convention devrait faire la nomenclature de critères ou seuils, techniques et socio-économiques $^{89}$, permettant de considérer qu'une altération du milieu ambiant constitue une modification lente de l'environnement qui ouvre la porte à la migration définitive de citoyens. Des critères ou seuils spécifiques s'appliqueraient à différentes situations de modifications lentes de l'environnement : changements climatiques, désertification, salinisation des terres cultivables, érosion des sols, pénuries d'eau potable, etc. Deux listes d'États seraient annexées à la convention. L'une énumérerait ceux qui acceptent de recevoir des migrants sur une base permanente et l'autre mentionnerait les États qui se déclarent ouverts à l'émigration de leurs ressortissants ${ }^{90}$.

La convention devrait prévoir des modalités spécifiques afin de compléter les conventions régionales applicables aux migrations environnementales. Mentionnons, à titre d'exemple, la situation particulière du continent africain.

L'Organisation pour l'unité africaine ${ }^{91}$ a amendé la Convention de l'OUA de $1969^{92}$, afin d'y intégrer des dispositions pour porter assistance au

88. Voir G.S. McCue, supra note 16 à la p. 179.

89. En ce qui concerne les seuils techniques, il peut être mentionné, à titre d'exemple, les concentrations de différents polluants atmosphériques et la disponibilité de quantités déterminées d'eau potable par habitant. Pour les critères socio-économiques, des exemples pertinents seraient un pourcentage de diminution de la source de revenu d'un travailleur et un niveau plancher de fourniture de services sociaux de base : éducation, santé, etc.

90. À l'image du système généralisé de préférence (S.G.P.) en matière commerciale : Q.D. Nguyen et al., supra note 15 aux pp. 1123-26.

91. À laquelle a succédé l'Union africaine, le 11 juillet 2000, au sommet de Lomé. L'Union africaine regroupe 53 États africains, sauf le Maroc; A. Kiss et J.-P. Beurier, supra note 56 à la p. 99. 
grand nombre de personnes déplacées par les guerres d'indépendance des années 60 qui sévissaient dans toute l'Afrique. Les migrations avaient comme origine la destruction des moyens de subsistance des populations, notamment les infrastructures économiques et sociales indispensables, comme les puits et systèmes d'approvisionnement d'eau potable et d'irrigation. Leur indisponibilité occasionna des famines. L'OUA adopta une définition plus large du terme réfugié, afin de solutionner les problématiques régionales non couvertes par la Convention de 1951. Elle étendit aux personnes déplacées par les perturbations du milieu, la portée du terme réfugié de la Convention de $1951^{93}$.

La Convention de l'OUA reprend la définition de réfugié de la Convention de 1951 et y ajoute un deuxième paragraphe :

Le terme «réfugié»s'applique également à toute personne qui, du fait d'une agression, d'une occupation extérieure, d'une domination étrangère ou d'événements troublant gravement l'ordre public dans une partie ou dans la totalité de son pays d'origine ou du pays dont elle a la nationalité, est obligée de quitter sa résidence habituelle pour chercher refuge dans un autre endroit à l'extérieur de son pays d'origine ou du pays dont elle a la nationalité $e^{94}$ (notre soulignement).

Cette définition inclut sans contredit les réfugiés environnementaux. En effet, lorsque des personnes fuient à cause d'une dégradation de l'environnement, elles cherchent refuge d'un «événement qui trouble gravement l'ordre public» ${ }^{95}$.

Dans le système établi par la Convention de l'OUA, les réfugiés qui rencontrent seulement les conditions de la deuxième partie de la définition et

92. Convention de l'OUA, supra note 11.

93. Tel est aussi le cas de la Déclaration de Cartagena sur les réfugiés, 22 novembre 1984, OAS/Ser.L./V/II.66, doc. 10, rev. 1 aux pp. 190-93. La Déclaration de Cartagena a été adoptée afin d'assister les deux millions de réfugiés issus de 10 années de guerre en Amérique centrale. Elle s'est inspirée de la définition de réfugié que l'on retrouve dans la Convention sur l'OUA.

94. Convention de l'OUA, supra note 11, art. 1 (2).

95. Ibid., art. 1(2). 
non celles découlant de la Convention de 1951, reçoivent une protection exclusivement temporaire. ${ }^{96}$ Bien que ces réfugiés ne soient pas forcés de retourner dans leur État d'origine, ils ne peuvent pas s'établir, à long terme, dans l'État d'accueil. Ceci s'explique par le fait que les réfugiés africains, délocalisés par les guerres d'indépendance, ne requéraient qu'une protection passagère. Lorsque les conflits de libération prenaient fin, les personnes pouvaient retourner chez elles ${ }^{97}$.

La convention sur les migrants environnementaux compléterait la Convention de l'OUA de 1969, en ce qu'elle encadrerait le sort des migrants, victimes des modifications lentes de l'environnement. Ces derniers cherchent un refuge définitif à la suite d'une importante dégradation de l'environnement. Ils requièrent, par conséquent, une protection prolongée, ce que ne leur offre pas la Convention de l'OUA.

La Convention de l'OUA serait inadaptée aux importantes problématiques que constituent, pour le continent africain, les modifications lentes de l'environnement que sont la désertification et la sécheresse. Cette lacune fait dire à un auteur que la Convention de l'OUA est inefficace «to provide environmental refugees with the protection that they need to survive» ${ }^{98}$. Une convention sur les migrations environnementales pourrait pallier à cette lacune de la Convention de l'OUA, en prévoyant des mécanismes permettant l'établissement définitif des populations qui en sont victimes.

\section{b) Les principes sous-jacents à la convention, selon Gregory S. McCue $^{99}$}

Une convention spécifiquement consacrée aux migrations environnementales pourrait s'articuler autour des principes qui découlent de

96. Carlos Ortiz Miranda, «Toward a Broader definition of refugee: 20th Century Development Trends» (1990) 20 Cal. W. Int'l L.J. 315, aux pp. 325-26.

97. J. B. Cooper, supra note 5 à la p. 498.

98. Ibid. à la p. 499.

99. L'idée d'élaborer une convention à partir des principes qui découlent de l'article 21 de la Déclaration de Stockholm sur l'environnement, comme il sera exposé, est attribuable à G. S. McCue, supra note 16. La sous-section b) résume la portion de l'article de l'auteur, à ce sujet. 
l'article 21 de la Déclaration de Stockholm sur l'environnement ${ }^{100}$, qui constituent les fondements du droit international de l'environnement ${ }^{101}$. Il s'agit : du devoir de prévention, du devoir de minimiser les dommages et de procurer assistance, qui inclut : la notification, la fourniture d'information et le développement de plans de contingence, du devoir de coopération et du devoir de compensation.

L'apport de chacun de ces principes dans l'élaboration d'une convention portant sur les réfugiés environnementaux est envisagé sommairement.

En ce qui concerne le devoir de prévention ${ }^{102}$, les États devraient tenir compte dans l'élaboration de leurs politiques de développement économique, notamment au niveau industriel, des risques d'accidents et de dégradation à long terme de l'environnement.

Lorsque la prévention ne déboucherait pas sur des résultats positifs, l'État dans lequel la catastrophe environnementale se produirait aurait l'obligation de minimiser les dommages et de fournir une assistance aux victimes. Ces obligations incomberaient aussi aux autres États. Ce devoir, comme il a été mentionné, se décompose en trois volets : la notification, la fourniture d'information et le développement de plans de contingence. En ce qui concerne le premier $^{103}$, il consisterait à informer, d'une part, les États voisins susceptibles d'être affectés par le flot de migrants et, d'autre part, le secrétariat de la convention. Le devoir de fournir de l'information ${ }^{104}$ impliquerait, quant à lui, de rendre disponible les données précises relatives à l'accident. Celles-ci seraient requises, particulièrement pour les États intéressés à fournir de l'aide, puisqu'il serait nécessaire qu'ils soient adéquatement informés de la situation à laquelle ils sont confrontés. Le devoir de développer des plans de contingence ${ }^{105}$ viserait à anticiper les solutions aux problèmes causés par les migrations de populations. Les plans de contingence détaillés devraient être communiqués au secrétariat de la

100. Déclaration de Stockholm sur l'environnement, supra note 66.

101. G. S. McCue, supra à la p. 179.

102. Ibid. aux pp. 180-182.

103. Ibid. aux pp. 182.

104. Ibid. aux pp. 183-84.

105. Ibid. aux pp. 184-85. 
convention, en vertu des devoirs de notification et d'information. Les États voisins de celui auquel le plan de contingence s'appliquerait, devraient être impliqués dans l'élaboration de ce dernier.

Le devoir de coopération ${ }^{106}$ prendrait une signification différente pour les situations de modifications lentes de l'environnement et pour celles des catastrophes à proprement parler. Quelques commentaires s'imposent en ce qui concerne les premières. Le devoir de coopération s'articulerait, dans ce cas, autour de mesures visant à simplifier la migration internationale définitive des personnes. Les parties signataires de la convention s'engageraient à développer des modalités facilitant l'établissement des migrants sur leur territoire. Les États pourraient, par exemple, prévoir l'accélération du processus de traitement des demandes afin d'obtenir le statut d'immigrant ou de réfugié ${ }^{107}$. Les États développés s'engageraient à négocier avec des États en développement, victimes de modifications lentes de l'environnement, les modalités de l'établissement graduel des personnes dans l'État d'accueil, selon un échéancier prévoyant le transfert d'un certain nombre de personnes. L'entente qui pourrait être conclue entre l'Australie ou la Nouvelle-Zélande et le Tuvalu, victime des changements climatiques, serait susceptible de servir de modèle pour le futur ${ }^{108}$.

Le devoir de compensation pour des pertes résultant de dommages environnementaux ${ }^{109}$ signifierait que les États qui enfreindraient l'un des devoirs mentionnés engageraient leur responsabilité et devraient, par conséquent, indemniser les États victimes de migrations environnementales. Puisque les migrations résultent de causes anthropiques, mais aussi naturelles, le régime de responsabilité adopté devrait se fonder sur la responsabilité sans faute. Un fonds d'indemnisation des États victimes de migrations environnementales pourrait être créé.

106. Principe 24 de la Déclaration de Stockholm sur l'environnement, supra note 66. Consulter à ce sujet A. Kiss et J.-P. Beurier, supra note 56 aux pp. 127-29.

107. Supra note 72.

108. En ligne : <http ://www.guardian.co.uk/globalwarming/story/ 0,7369,651076,00.html> (5 janvier 2006); en ligne : <http://webdiary.smh.com.au/ archives/margo_kingston_ comment/000686.html> (5 janvier 2006).

109. G. S. McCue, supra note 16 aux pp. 185-86. 
À la recherche d'un statut juridique pour

les migrants environnementaux transfrontaliers : $\quad$ (2006) 37 R.D.U.S.

la problématique de la notion de réfugié

La convention indiquerait que les États sont responsables des migrations consécutives aux modifications environnementales. Les États endosseraient sans difficulté cette responsabilité car «in the narrow context of environmental migration, it seeems that the consequences of inaction are serious enough and the extension of responsabilities narrow enough to override state trepidation $\gg{ }^{110}$.

Les principes énoncés permettraient de lever les obstacles pratiques et politiques à la résolution du problème des migrations environnementales ${ }^{111}$. Les pays en développement appuieraient plus facilement l'adoption de la convention que les pays développés qui partagent, dans ce cas, des craintes identiques à celles mentionnées à la section $\mathrm{A})^{112}$.

\section{Conclusion}

La conjoncture internationale n'est pas propice à l'élaboration d'une stratégie de gestion des migrations environnementales. En effet, la lutte antiterroriste est une composante importante, sinon centrale, de la politique étrangère des États. La conséquence de cette situation pourrait être la suivante : puisque la définition de réfugiés revient ultimement à l'État, ce dernier aura tendance à qualifier les flots migratoires en fonction de sa volonté de donner ou non l'asile à des personnes en accordant une importance disproportionnée à la question du terrorisme international. Ainsi, en qualifiant une personne de réfugié économique, plutôt que politique, un gouvernement lui ferme la porte ${ }^{113}$.

C'est dans ce contexte qu'il faut situer l'attitude générale des États sur la question des réfugiés environnementaux, qui est influencée à des degrés divers par la position du gouvernement américain relativement à la lutte antiterroriste. Cette dernière sert d'argument aux dirigeants américains afin de défendre auprès de l'électorat, la conception selon laquelle les critères de sélection des étrangers autorisés à franchir la frontière états-unienne doivent

\footnotetext{
110. Ibid. aux pp. 180 .

111. Ibid. aux pp. 179.

112. Ces derniers craignent un flot de réfugiés traversant leurs frontières. Ils craignent aussi de devoir supporter des coûts substantiels découlant de l'application de la convention.

113. Voir P. Le Prestre, supra note 20 à la p. 409.
} 
À la recherche d'un statut juridique pour
(2006) 37 R.D.U.S. $\quad \begin{gathered}\text { les migrants environnementaux transfrontaliers } \\ \text { la problématique de la notion de réfugié }\end{gathered}$

être compatibles avec la stratégie gouvernementale de lutte au terrorisme. La position américaine sur les changements climatiques n'aide de plus en rien à l'élaboration d'une stratégie internationale sur les migrations environnementales.

Bien que certains mécanismes internationaux aient été institués afin de venir en aide aux victimes d'altérations du milieu ambiant, notamment le HCNUR, ces derniers doivent être constamment améliorés. Par exemple, il a été suggéré, le 23 juin 2005, lors d'un colloque portant sur les Réfugiés environnementaux, tenu à Limoges en France, de créer une Agence internationale de «casques verts» représentée dans chaque région du monde afin de venir en aide aux personnes déplacées ${ }^{114}$. Cette idée, si elle était appliquée, optimiserait la coordination des opérations de secours de l'organisme sur le terrain.

La spoliation de l'environnement oblige les États à définir des cadres normatifs afin d'agir a posteriori, mais aussi a priori. C'est également sous ce double aspect que doit être envisagée la gestion des migrations environnementales.

L'importance du problème des migrations environnementales a été soulignée par de nombreux gouvernements des pays développés depuis le début des années 90 et ces derniers savent qu'ils ne peuvent continuer d'exercer la politique de l'autruche.

114. Faculté de droit de Limoges, «Appel de Limoges sur les réfugiés écologiques» (23 juin 2005) en ligne : <http://www.fdse.unilim.fr/>. 